\title{
Quasiperiodic photonic crystal microcavity lasers
}

\author{
Kengo Nozaki and Toshihiko Baba ${ }^{\text {a) }}$ \\ Yokohama National University, Department of Electrical and Computer Engineering, 79-5 Tokiwadai, \\ Hodogayaku, Yokohama 240-8501, Japan
}

(Received 26 January 2004; accepted 1 April 2004; published online 25 May 2004)

\begin{abstract}
We fabricated a 12-fold symmetric quasiperiodic photonic crystal (QPC) point defect laser, and obtained the lasing action at room temperature. When photopumping a seven-hole-missing defect or a uniform QPC part, the lasing occurred by different modes. The finite-difference time-domain analysis showed that the defect mode is a whispering gallery mode, which is confined by the photonic gap or the boundary reflection. It also indicated that the defect-free mode is an extended but partly localized mode, which cannot be seen in photonic crystals. (C) 2004 American Institute of Physics. [DOI: 10.1063/1.1762705]
\end{abstract}

The semiconductor photonic crystal (PC) has a translationally symmetric periodicity and exhibits a photonic band gap (PBG), which enables us to form a microcavity laser by introducing a point defect. ${ }^{1-3}$ Even when without point defects, lasing is also possible by the distributed feedback of light at a photonic band edge. ${ }^{4-6}$ These phenomena are based on light propagation as Bloch waves in the PC. On the other hand, the quasiperiodic PC (QPC) has high-order rotational and line symmetries. ${ }^{7-11}$ Therefore, it should not have propagation characteristics of Bloch waves, but exhibit unique characteristics of the mixture of light propagation and localization. For example, a photonic gap like a PBG in PCs was demonstrated in Penrose-type $\mathrm{QPCs}^{7-9}$ and a 12-fold symmetric QPC composed of square and equilateral triangular basis. ${ }^{10}$ In Penrose QPCs, light behavior inside the point and line defects have been studied, and the lasing has been demonstrated in an organic material QPC without defects. ${ }^{11}$ In our previous work, we theoretically discussed a microcavity in a 12-fold symmetric QPC composed of holes arranged in a semiconductor membrane. ${ }^{12}$ Through the threedimensional finite-difference time-domain (FDTD) analysis, we estimated that a seven-hole-missing point defect has high structural consistency with a whispering gallery mode (WGM) and achieves a much higher $Q$ than in a microdisk ${ }^{13}$ and a microgear. ${ }^{14}$ In this letter, we demonstrate the lasing action in the QPC point-defect microlaser. The lasing was observed not only inside but also outside of the defect. We show the correspondence of the experimental result with the FDTD analysis, and discuss unique properties, which are different from PCs.

In the experiment, a GaInAsP-InP epitaxial wafer with a photoluminescence peak wavelength of $1.58 \mu \mathrm{m}$ was prepared. The GaInAsP active layer of $\sim 0.24 \mu \mathrm{m}$ in total thickness was composed of five compressively strained quantum wells and separate confinement heterostructure layers. The fabrication process was the same as described in Ref. 15; holes were formed by electron-beam lithography and $\mathrm{Cl}_{2} / \mathrm{Xe}$ inductively coupled plasma etching, and the membrane structure was formed by $\mathrm{HCl}$ wet etching. Figure 1 shows the top view of a device. The seven-hole-missing point defect

was placed at the center and 11-13 rows of holes (a total of 516 holes) were surrounding it. The sidewall roughness of the holes was $\sim 20 \mathrm{~nm}$. In the measurement, each device was photopumped by pulsed laser light at $\lambda=0.98 \mu \mathrm{m}$ with a duty ratio of $0.075 \%$ and a focused spot diameter of 5-6 $\mu \mathrm{m}$ at room temperature (293-295 K). Emitted light from the device was detected by a multimode fiber and analyzed by optical spectrum analyzer. The lasing action was observed in many devices with hole diameter $2 r$ of $0.410-0.490 \mu \mathrm{m}$ and lattice constant $a$ of $0.550-0.665 \mu \mathrm{m}$. These parameters are slightly larger than those of normal PCs. Figure 2(a) shows the lasing characteristic, which exhibited the lowest threshold, when the point defect was mainly excited, as shown in the inset. The threshold pulse peak power of light irradiation was $0.8 \mathrm{~mW}$. This value is lower than $1.0-1.4 \mathrm{~mW}$ for a similar seven-hole-missing defect in a triangular lattice PC fabricated and measured in the same way. Above threshold, the clear single-mode spectrum was observed at $\lambda \sim 1.60 \mu \mathrm{m}$. The mode peak at 2.6 times the threshold was $20 \mathrm{~dB}$ higher than the background spontaneous emission level. On the other hand, clear single-mode lasing was also observed, when the uniform QPC part outside of the defect was excited, as shown in Fig. 2(b). The threshold power of the defect-free mode lasing was almost the same as the defect mode lasing.

To explain lasing modes, we performed the twodimensional (2D) FDTD analysis with the equivalent index approximation. First, we calculated the transmission spec-

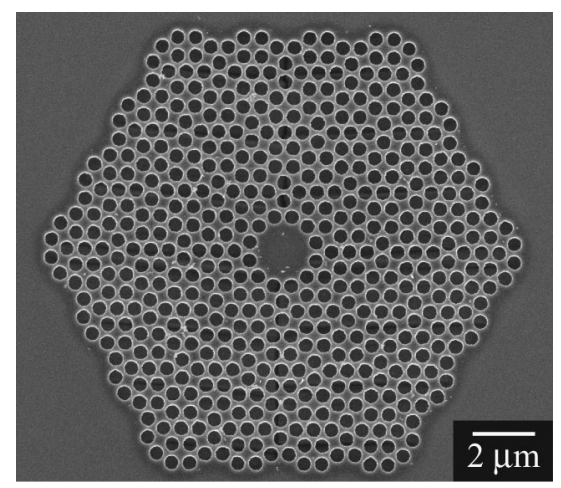

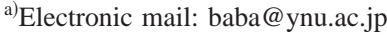

FIG. 1. Top view of fabricated 12-fold symmetric QPC point defect laser. 


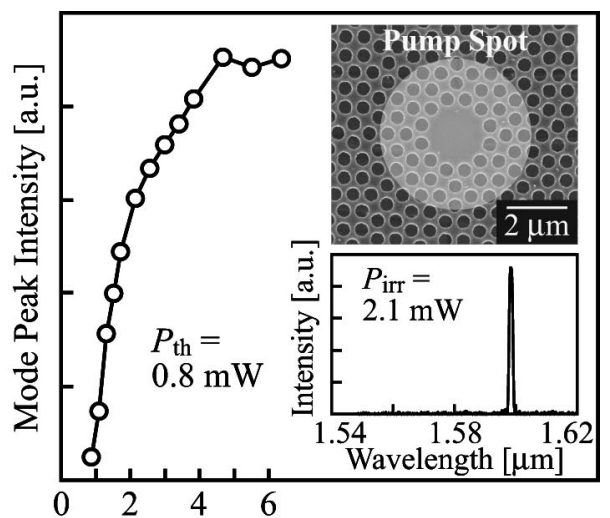

Irradiated Power $P_{\text {irr }}[\mathrm{mW}]$

(a)

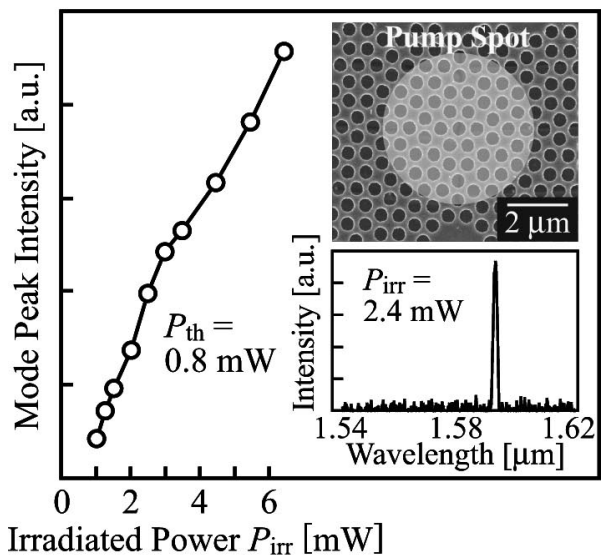

(b)

FIG. 2. Lasing characteristics observed by photopumping at room temperature. (a) Defect mode lasing for $2 r=0.435 \mu \mathrm{m}$ and $a=0.610 \mu \mathrm{m}$. (b) Defect-free mode lasing for $2 r=0.415 \mu \mathrm{m}$ and $a=0.635 \mu \mathrm{m}$.

trum of the QPC to confirm the photonic gap. Here, a rectangular QPC with $\sim 20$ rows of holes in the direction of light transmission (a total of 337 holes) was modeled. The equivalent index of the membrane was assumed to be 2.73. A Gaussian pulse excitation was given for the polarization inside the 2D plane with a center normalized frequency $a / \lambda_{0}$ of 0.366 and a full spectral width at $1 / e^{2}$ of the maximum intensity of $0.4\left(2 \pi c / \lambda_{0}\right)$, respectively, where $c$ is the light velocity in a vacuum. Figure 3 shows transmission spectra calculated for $2 r / a=0.769$. Against two different incident

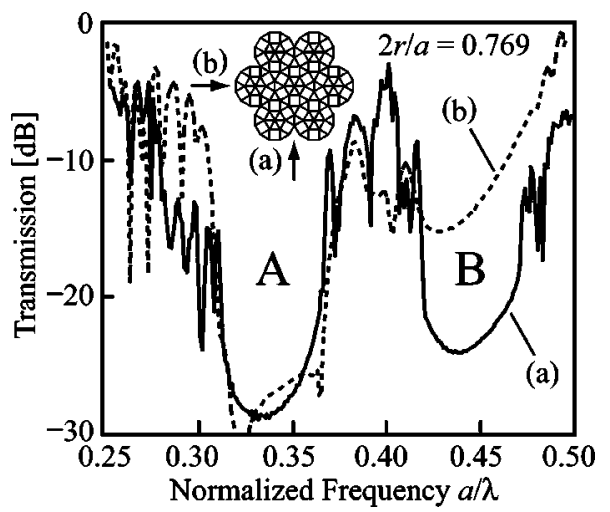

FIG. 3. Transmission spectra of QPC with $2 r / a=0.769$ calculated by $2 \mathrm{D}$ FDTD method. Inset shows a part of the QPC structure and different light

shown in Fig. 4. Calculated field distributions of modes at
Downloaded 30 May 2004 to 133.34.23.211. Redistribution subject to AlP license or copyright, see http://apl.aip.org/apl/copyright.jsp

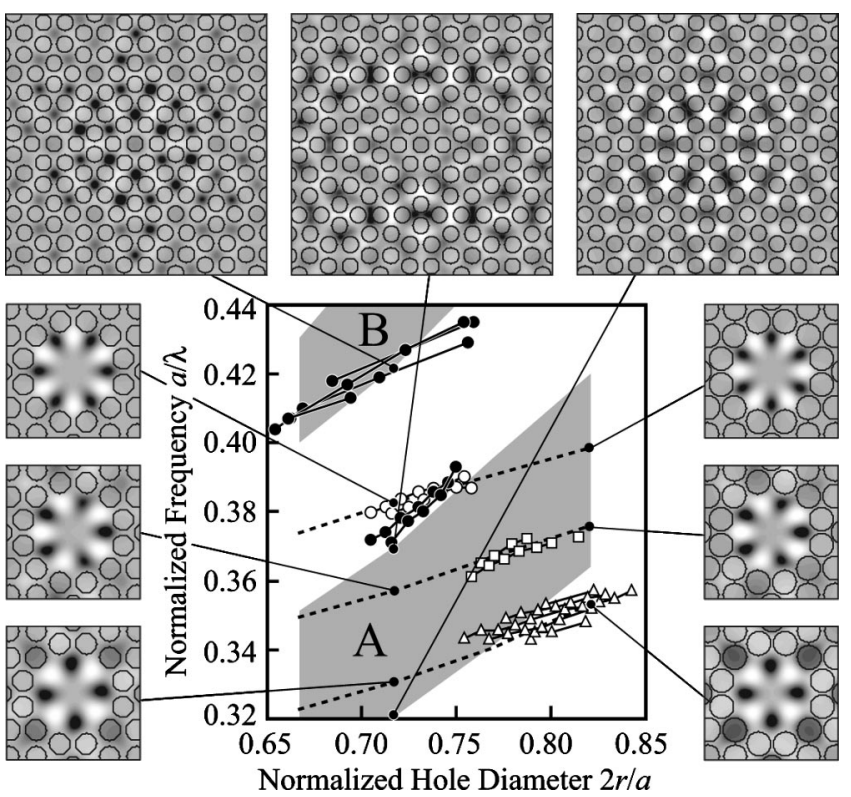

FIG. 4. Mode frequency shift vs normalized hole diameter characteristics. White and black plots with solid lines are experimental results for defect mode and defect-free mode lasing, respectively. Dashed lines and field distributions are calculated ones. Gray area indicates calculated photonic gaps of A and B in Fig. 3. Here, calculated results are slightly tuned for the best fitting to experimental results.

angles (a) and (b), the transmission is strongly suppressed in frequency ranges $\mathrm{A}$ and $\mathrm{B}$. In particular, a transmission suppression of $\sim 30 \mathrm{~dB}$ is observed in frequency range $\mathrm{A}$ for both incident angles. Therefore, this range can be thought as a photonic gap of the structure.

Figure 4 summarizes the normalized frequency $a / \lambda$ of lasing modes with the normalized hole diameter $2 r / a$ for all samples. Here, white and black plots with solid lines show experimental results for the defect mode and defect-free mode lasing, respectively. Dashed lines and mode field (magnetic field normal to the 2D plane) distributions show calculated results with a cavity model very similar to that in the experiment. The gray area indicates the photonic gap of frequency range A and B in Fig. 3. Regarding the defect mode, the frequency shift with $2 r / a$ is in good agreement with the experimental one. Three modes observed for different lattice constants $a$ are WGMs having different azimuthal orders, as understood from calculated field distributions. The lowest threshold was recorded for the highest $a / \lambda$ mode, whose number of standing wave matches with the number of innermost holes. This result suggests that high structural consistency between the mode profile and the structure is important, as discussed for microgears. ${ }^{14}$ Note that their frequencies are not limited inside the photonic gap but continue to the outside of the gap. This suggests that defect modes are confined not only by the photonic gap but also by total internal reflection or partial reflection arising from the mode mismatching at the boundary between the defect and the QPC. This discussion is supported by the frequency shift line of the defect mode crossing with the defect-free mode line discussed below.

Regarding the defect-free mode, the slope of the frequency shift in the experiment corresponds well to the slope of the top edge of gap A and the bottom edge of gap B, as shown in Fig. 4. Calculated field distributions of modes at AIP license or copyright, see http://apl.aip.org/apl/copyright.jsp 
these gap edges are widely extending over the QPC, even though the QPC has no translational symmetries. This is because the structure studied here has many similarities, compared with, e.g., Penrose type. Note that field maxima of these modes are concentrating inbetween holes. It is similar to distributed feedback modes in PCs. Still, they show some degree of localization, which can be lasing modes even against the partial excitation. Such unique characteristics cannot be seen in normal PCs and even in a low refractive index contrast QPC whose modes are dominated by lattice points. ${ }^{11}$

In conclusion, we fabricated a 12-fold symmetric QPC into a GaInAsP membrane and observed the roomtemperature lasing both inside and outside of a point defect. The FDTD calculation explained that the defect mode was confined by the photonic gap or the boundary reflection, and that the defect-free mode was a localized mode at the photonic gap edge. These modes can exist in the same device at the same frequency. This is very unique and cannot be seen in PC cavities. The defect mode demonstrated in this study was a WGM having a wide node at the center. Therefore, its $Q$ factor will not be significantly degraded even with center posts, which are effective for current injection and heat sinking under continuous-wave condition. Furthermore, lasing by other various modes, e.g., dipole modes in the single point defect, ${ }^{12}$ is also expected in the QPC. Because of the large structural flexibility, QPCs have the potential of becoming a nanocavity with higher $Q$ and smaller mode volume than those of PCs.
This work was supported by IT Program and 21st COE Program, both from the Ministry of Education, Culture, Sports, Science, and Technology. It was also supported by CREST of the Japan Science and Technology Corporation.

${ }^{1}$ O. Painter, R. K. Lee, A. Scherer, A. Yariv, J. D. O'Brien, P. D. Dapkus, and I. Kim, Science 284, 1819 (1999).

${ }^{2}$ H. Y. Ryu, S. H. Kim, H. G. Park, J. K. Hwang, Y. H. Lee, and J. S. Kim, Appl. Phys. Lett. 80, 3883 (2002).

${ }^{3}$ K. Inoshita and T. Baba, Electron. Lett. 39, 844 (2003).

${ }^{4}$ M. Imada, S. Noda, A. Chutinan, T. Tokuda, M. Murata, and G. Sasaki, Appl. Phys. Lett. 75, 316 (1999).

${ }^{5}$ C. Monat, C. Seassal, X. Letartre, P. Regreny, P. Rojo-Romeo, P. Viktorovitch, M. L. V. d'Yerville, D. Cassagne, J. P. Albert, E. Jalaguier, S. Pocas, and B. Aspar, Appl. Phys. Lett. 81, 5102 (2002).

${ }^{6}$ H. Y. Ryu, S. H. Kwon, Y. J. Lee, Y. H. Lee, and J. S. Kim, Appl. Phys. Lett. 80, 3476 (2002).

${ }^{7}$ Y. S. Chan, C. T. Chan, and Z. Y. Liu, Phys. Rev. Lett. 80, 956 (1998).

${ }^{8}$ C. Jin, B. Cheng, B. Man, Z. Li, D. Zhang, S. Ban, and B. Sun, Appl. Phys. Lett. 75, 1848 (1999)

${ }^{9}$ Y. Wang, X. Hu, X. Xu, B. Cheng, and D. Zhang, Phys. Rev. B 68, 165106 (2003).

${ }^{10}$ M. E. Zoorob, M. D. B. Charlton, G. J. Parker, J. J. Baumberg, and M. C. Netti, Nature (London) 404, 740 (2000).

${ }^{11}$ M. Notomi, H. Suzuki, T. Tamamura, and K. Edagawa, Phys. Rev. Lett. 92, 123906 (2004).

${ }^{12}$ K. Nozaki, A. Nakagawa, D. Sano, and T. Baba, IEEE J. Sel. Top. Quantum Electron. 9, 1355 (2003).

${ }^{13}$ M. Fujita, R. Ushigome, and T. Baba, Electron. Lett. 36, 790 (2000).

${ }^{14}$ M. Fujita and T. Baba, Appl. Phys. Lett. 80, 2051 (2002).

${ }^{15}$ K. Inoshita and T. Baba, IEEE J. Sel. Top. Quantum Electron. 9, 1347 (2003). 